\title{
Clinical Profile of Patients With Chronically Occluded Coronary Arteries: A Single Center Study
}

\author{
Vikrant Deshmukha, Mukund Vasantrao Phutane ${ }^{\mathrm{a}, \mathrm{b}}$, Kalyan Munde ${ }^{\mathrm{a}}$, \\ Narendra Bansal ${ }^{\mathrm{a}}$
}

\begin{abstract}
Background: A chronic total occlusion (CTO) is defined as an angiographically documented or clinically suspected complete interruption of antegrade coronary flow (Thrombolysis in Myocardial Infarction (TIMI)-0 flow) of greater than 3 months standing. Coronary CTOs represent the most technically challenging lesion subset that interventional cardiologists face. CTOs are identified in up to one third of patients referred for coronary angiography and remain seriously undertreated with percutaneous techniques. Decision to treat or not to treat a CTO is always confusing. This is an attempt to provide clinical profile of patients having totally occluded coronary arteries and their natural history.
\end{abstract}

Methods: The observational study was carried out in tertiary health center in Mumbai. Totally 117 patients who had CTO on angiography were selected. Their clinical presentation and angiography correlation was done and results were analyzed.

Results: Out of a total of 117 patients, $86(73.50 \%)$ were males, female $31(26 \%)$. All of the patients studied were above 40 years. Age group 40 - 49 years had $25(21.36 \%)$ patients, 50 - 59 years had 32 (27.35\%), 60 - 69 years had $43(36.75 \%),>70$ years had $17(14.52 \%)$ patients. Smoking as a risk factor was present in $32(27.35 \%)$, tobacco in $45(38.46 \%)$, alcohol in six (5\%), no addictions in $35(29.91 \%)$ patients. Diabetes in 63 (53.84\%), hypertension in 78 (66.67\%) patients, both were present in $49(41.88 \%)$, dyslipidemia in $37(31.62 \%)$ patients. Sixty-three $(53.84 \%)$ patients presented with unstable angina (UA)/non ST elevated myocardial infarction (NSTEMI), 32 (27.35\%) with chronic stable angina (CSA), ST elevated myocardial infarction (STEMI) in $22(18.80 \%)$. History of prior myocardial infarction (MI) was present in 36 (30.76\%), prior coronary artery bypass graft (CABG) in nine (7.6\%), prior percutaneous intervention (PCI) in $18(15.38 \%)$. Triple vessel disease (TVD) in 38 (32.47\%), double vessel disease (DVD) in 53 (45.29\%), single vessel disease (SVD) in $26(22.22 \%)$ patients. Left anterior descending artery (LAD) CTO was present in 40

Manuscript submitted July 15, 2018, accepted July 25, 2018

aDepartment of Cardiology, The Grant Medical College and Sir J.J. Group of Hospital, Mumbai, Maharashtra, India

${ }^{b}$ Corresponding Author: Mukund Vasantrao Phutane, Department of Cardiology, The Grant Medical College and Sir J.J. Group of Hospital, Byculla, Mumbai 400008, Maharashtra, India. Email: mak27remo@gmail.com

doi: https://doi.org/10.14740/cr743w
(34.18\%), right coronary artery (RCA) in $61(52.14 \%)$, left circumflex artery (LCX) obtuse marginal (OM) in $16(13.67 \%)$ patients.

Conclusions: Patients having CTO of coronary arteries are mostly above age of 40 years. Most common age group was 60 - 69 years. It was most common in males than females. Tobacco chewing was more commonly associated followed by smoking. Hypertension and diabetes were strongly associated with CTO. Most patients presented with unstable angina/NSTEMI followed by chronic stable angina. Old MI was present in one third of patients. Most common artery to be affected was RCA followed by LAD.

Keywords: CTO; Coronary artery disease; Clinical profile

\section{Introduction}

Chronic total occlusions (CTOs) are considered to be $100 \%$ coronary lesions, of more than 3-month evolution. They are commonly found in stable chronic patients, with varying levels of symptoms. After the culprit artery has been treated, patients with acute coronary syndrome may occasionally also have chronic occlusions in another artery that was not responsible for the acute event, and is therefore considered a CTO. CTO interventions were historically associated with a high rate of procedural complications. But with the improvement in technology and operator skills, the complication rate has come down. Several studies have demonstrated that successful CTO revascularization has translated into better cardiovascular outcomes and improved quality of life. World over presently about $70 \%$ of CTO interventions are successful. There are large studies on CTO interventions but lacking on the patient demographics and background, clinical presentations of CTO patients. This study planned to bridge this gap [1].

\section{Definition and incidence}

CTO is defined as complete interruption of antegrade coronary flow (Thrombolysis in Myocardial Infarction-0 (TIMI-0)) for more than 3 months. Those in the absence of recent symptom or symptoms due to other culprit vessel in patient with past angiogram showing total occlusion for more than 3-month duration can be assumed CTO. As the duration of lesion in- 
Table 1. Baseline Patient Profile

\begin{tabular}{|c|c|c|}
\hline & Number & Percentage (\%) \\
\hline \multicolumn{3}{|l|}{ Age } \\
\hline$<60$ & 57 & 48.71 \\
\hline$>60$ & 60 & 51.28 \\
\hline \multicolumn{3}{|l|}{ Gender } \\
\hline Male & 86 & 73.50 \\
\hline Female & 31 & 26.49 \\
\hline $\mathrm{DM}$ & 63 & 53.84 \\
\hline HTN & 78 & 66.67 \\
\hline Tobacco & 45 & 38.46 \\
\hline Smoking & 32 & 27.35 \\
\hline Dyslipidemia & 37 & 31.62 \\
\hline Previous PCI & 18 & 15.38 \\
\hline Previous CABG & 9 & 7.6 \\
\hline
\end{tabular}

DM: diabetes mellitus; HTN: hypertension.

creases there is development of collateral circulation, vessel distal to lesion filling retrogradely during angiogram. Bridging collaterals sometimes give a picture of antegrade flow distal to coronary occlusion but different cine angio angles distinguish the extra luminal course of these bridging collaterals. Intraluminal channels that are seen on histopathology facilitate wire crossing during procedure and by definition, have no continuity throughout the occluded segment.

\section{Prevalence and occlusion characteristics}

Incidence of CTO varies between 10 and $30 \%$ of all coronary angiograms depending upon type of population studied. Atypical symptoms misinterpreted at the time of the acute event account for the consistent persistence of CTOs in $18.4 \%$ of patients. Even STEMI patients had the incidence of $13 \%$, and these patients had poor immediate and long-term outcomes. If STEMI patients' coronary angiography CAG shows CTO in a non-infarct related artery then CTO is independent predictor for both early mortality and late mortality. During any situation if there is hampering of blood flow to collaterals to the occluded vessel or acute impairment of preexisting collaterals from the acutely occluded vessel to the CTO jeopardizing a large myocardial territory, which also explains the prognostic benefit of reanalyzing CTOs.

Lesion characteristics play an important role in the likelihood of a successful recanalization. Morino et al introduced a lesion-related difficulty grading tool, the J-CTO score, based on a large series of anterograde recanalization in Japan [2]: 1) CTO length greater or lesser than $20 \mathrm{~mm}$; 2) Presence of a greater than 45 degrees bend within the occlusion; 3) Presence of intralesional calcification; 4) Entry shape tapered or blunt; 5) Previous failed attempt.

Lesions with J-CTO score of 0 - 1 had high success rate $>$ $90 \%$ and difficult lesion labeled for J-CTO score $\geq 3$, had 73.3
Table 2. Presenting History of CTO Patient

\begin{tabular}{lll}
\hline & Number & Percentage (\%) \\
\hline STEMI & 22 & 18.80 \\
UA/NSTEMI & 63 & 53.84 \\
CSA & 32 & 27.35 \\
\hline
\end{tabular}

$\%$ success rate and need prolonged time for crossing. Technical progress and the introduction of the retrograde approach have certainly modified these percentages. Non-invasive imaging, in particular coronary multidetector computed tomography (MDCT), can help delineate the characteristics of the CTO. With coronary MDCT the occluded segment can be better delineated, calcium more reliably detected and quantified, the tortuosity and vessel path followed, the true length of the lesion better defined [3].

\section{Materials and Methods}

It was an observational study of all patients undergoing $\mathrm{CAG}$ as elective or emergency, patients who had CTO on angiography were selected. Study was conducted in the Department of Cardiology Government Medical College Mumbai, from 2016 to March, 2018.

\section{Inclusion criteria}

Patients undergoing CAG who had CTO on angiography were selected. CTO is defined as a high-grade coronary occlusion with TIMI grade 0 flow with estimated duration of at least 3 months.

\section{Exclusion criteria}

Exclusion criteria included patients with coronary total occlusion duration less than 3 months.

\section{Results}

\section{Baseline patient characteristics}

A total of 117 CTO patients were noted during the study period from 2016 to March, 2018. The mean age was 56 years (Table 1).

\section{Baseline cardiac profile}

In the study $63(53.84 \%)$ presented with UA/NSTEMI, 32 $(27.35 \%)$ with chronic stable angina, STEMI in $22(18.80 \%)$. Majority of the patients were in New York Heart Association (NYHA) functional class (FC) II (Table 2). 
Table 3. Coronary Involvement in CTO

\begin{tabular}{|c|c|c|}
\hline & Frequency & Percentage $(\%)$ \\
\hline \multicolumn{3}{|c|}{ Number of vessel involved } \\
\hline 1 (SVD) & 26 & 22.22 \\
\hline 2 (DVD) & 53 & 45.29 \\
\hline 3 (TVD) & 38 & 32.47 \\
\hline \multicolumn{3}{|c|}{ Target vessel involved } \\
\hline LAD & 40 & 34.18 \\
\hline LCX & 16 & 13.67 \\
\hline $\mathrm{RCA}$ & 61 & 52.14 \\
\hline
\end{tabular}

SVD: single vessel disease; DVD: double vessel disease; TVD: triple vessel disease.

\section{Coronary artery involvement}

Left anterior descending coronary artery (LAD) was involved in $34.18 \%(\mathrm{n}=40)$, right coronary artery $(\mathrm{RCA})$ in $51.28 \%(\mathrm{n}$ $=61)$, and left circumflex coronary artery (LCX) in $13.67 \%$ $(n=16)$. Coronary involvement in CTO is shown in Table 3.

\section{One case report}

We would like to represent a case of a 68-year-old man who was hypertensive, tobacco chewer presented with chest pain and dyspnea on exertion since 6 months ago with baseline electrocardiogram (ECG) within normal limit (Fig. 1), no significant finding on chest X-ray (Fig. 2); but had positive stress imaging, so we had coronary catheterization of this patient which revealed mid RCA total occlusion and distal RCA filling retrogradely from left coronary artery (Fig. 3).

\section{Discussion}

\section{Rationale and indications to CTO recanalization}

Although revascularization surgery is the most frequent treatment, clinicians and invasive cardiologists often consider the need and feasibility of percutaneous treatment for these lesions based on symptoms and prognostic factors. There are also significant differences in the treatment of CTO with geographical area and different centers in same geographic area. These differences can only be justified by some generally ill-defined indications and technical difficulty, which means that not all invasive cardiologists can or should deal with complex lesions of this type. The indications for percutaneous treatment of CTO are not well defined for treatment of CTO. Only the American guidelines for the appropriate use of percutaneous coronary treatment contain a clear position on treatment that is appropriate, uncertain or not indicated in CTO lesions.

Relief of symptomatic ischemia, angina and improvement of prognosis are the ultimate goals of CTO revascularization. Successful CTO percutaneous coronary intervention (PCI) improves angina and quality of life, improves left ventricular function, exercise tolerance, decreased need for CABG and decreased cardiac mortality. Decision to revascularize a CTO heavily depends upon J-CTO score and the presence of other critically diseased arteries. Viability in the territory supplied by the occluded vessel, angina symptoms or angina equivalents should be confirmed before taking decision to revascularization.

MRI imaging techniques are most suitable to define viability and ischemia. Late gadolinium enhancement less than $50 \%$ of wall thickness with MRI and reversible perfusion deficit more than $10 \%$ of the total myocardial mass with myocardial nuclear perfusion scan are gold standards for viability and ischemia. Patients with poorly controlled angina symptoms with medical therapy may also have indications to revascularization. Secondary causes of angina, such as anemia or hyperthyroidism must be appropriately corrected. CABG should be considered in the presence of left main coronary artery disease, complex triple vessel disease (especially in patients with diabetes, severe LV dysfunction or chronic renal insufficiency), occluded proximal LAD and multiple CTOs with high J-CTO score, rest for PCI. Final decision should be team approach among clinicians, interventionists and cardiac surgeons. Data from large national registries (British Cardiovascular Interventional Society (BCIS) suggest underutilization of PCI for CTO, and far below its prevalence. The preference given to surgery is probably not justified because recent trials show that more than $30 \%$ (in some series in excess of $50 \%$ ) of CTOs initially scheduled for CABG were not grafted because of poor

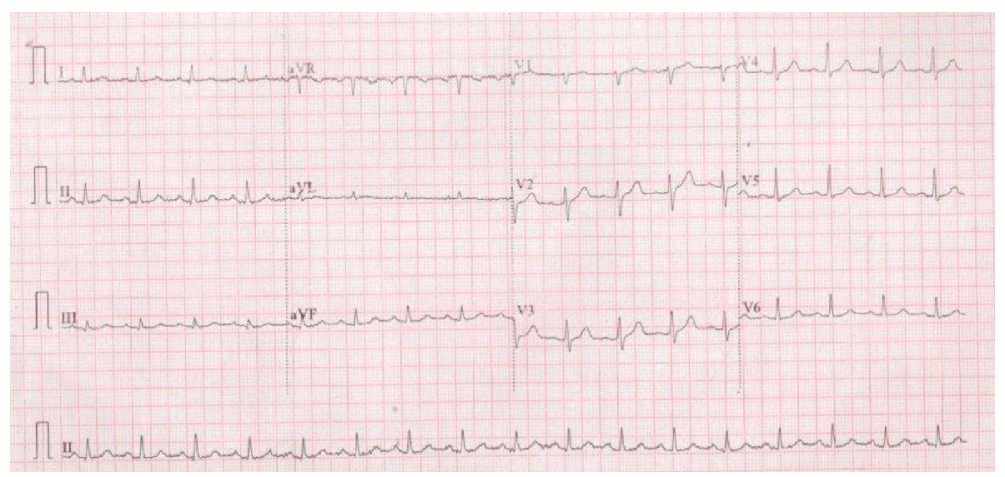

Figure 1. ECG of the patient with chronic stable angina showing sinus tachycardia, normal axis with no significant ST-T changes. 


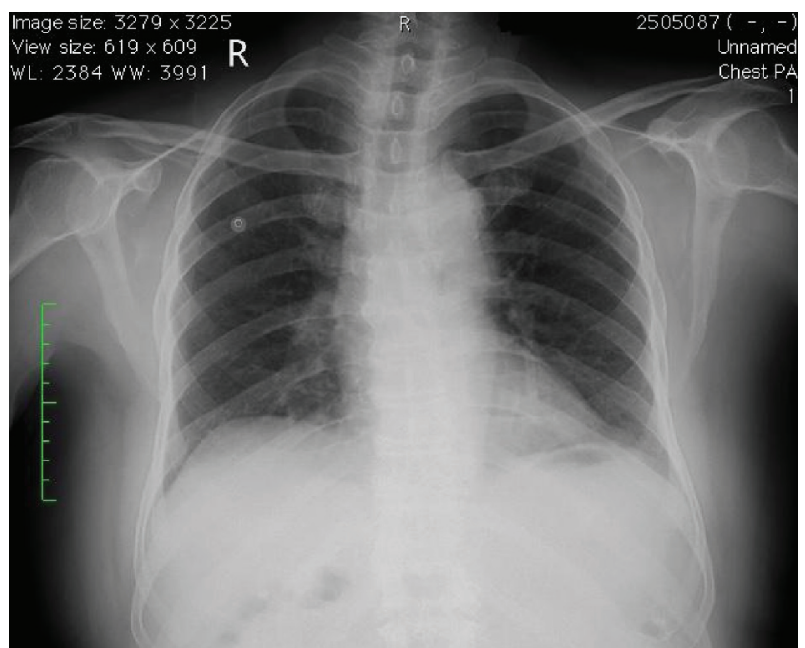

Figure 2. Chest X-ray PA view of the same patient with chronic stable angina showing prominent aortic knuckle, rest within normal limit.

target vessel and high rate of vein graft occlusion (the most frequently used conduits for right and left circumflex coronary arteries) [4].

\section{Demographic and clinical profile of coronary CTO patients}

The true prevalence of a CTO in the general population is unknown and not well studied. In a few older studies the prevalence of CTO in patients undergoing coronary angiography ranged between $10 \%$ and $52 \%$ depending on the clinical profile of the patient being examined. Among patients who underwent coronary angiography because of suspected coronary artery disease, CTO prevalence was $18 \%$ [5]. CTO prevalence was observed to be higher (54\%) among patients with a history of CABG surgery and lower (10\%) among patients with acute coronary syndrome undergoing primary PCI [6]. Several studies showed that CTO patients are a high-risk population with more traditional cardiovascular risk factors, multivessel disease, history of myocardial infarction (MI) and PCI [7].
A majority of patients in this study were middle aged. The mean age was 56 . In a comparative large Italian study the mean age was 62 [8]. Twenty-six percent of patients were women and $74 \%$ were men, compare to $80 \%$ male in Latvia study, this is similar to the trend seen globally $[4,8]$.

In our study higher proportion of patients was tobacco chewers $(38 \%)$ and $27 \%$ were smokers, compared to $42 \%$ in European study. This shows tobacco chewing as significant risk trend in Indian population. Diabetes is a very strong emerging risk factor found in $53 \%$ vs. $19 \%$, hypertension $66 \%$ vs. $83 \%$, dyslipidemia in $31 \%$ vs. $69 \%$ compared to National Cardiovascular Registry of the United States (NCDR).In CTO, RCA is the most common vessel affected followed by LAD and LCX. In our study history of prior MI was present in 30\%, prior CABG in $7.6 \%$, prior PCI in $15.38 \%$ compared to $73 \%$, $8.5 \%$ and $51.3 \%$ respectively in NCDR study [9].

Data from registries reveal striking variability for CTO percutaneous intervention between different centers and countries. For example, in Japanese centers CTO incidence was $19 \%$, and $61.2 \%$ of these cases were treated percutaneously [10]; however in North America, where CTOs are more frequent $(29-33 \%)$, only between $6 \%$ and $9 \%$ of patients were treated percutaneously [11].

According to the current European Society of Cardiology (ESC) revascularization guidelines, CTO percutaneous revascularization is considered in patients with myocardial ischemia given myocardial segment and/or symptoms associated with angina (IIA, class B level recommendation). A retrograde approach is recommended as a class $\mathrm{IIb}$ recommendation level $\mathrm{C}$, after an unsuccessful antegrade approach or as a starting method for selected patients [12].

\section{Conclusions}

This prospective CTO study shows predominantly male population had coronary CTOs. The incidence of tobacco chewing and smoking is high and might have contributed to the problem. Malignant atherosclerotic tendency in Indian population calls for population preventive strategies. Patients with CTO
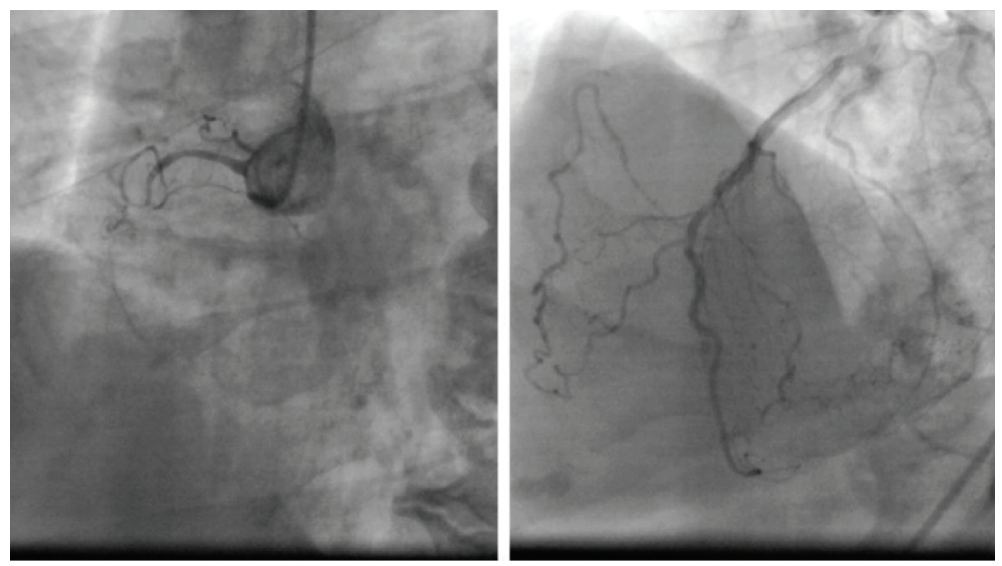

Figure 3. Coronary angiography of the same patient showing chronic right coronary artery total occlusion with left coronary shoot revealing retrograde flow to RCA. 
have a clinical profile that is different to that of patients with other coronary ischemic disease in general. These differences are not only in terms of greater severity of coronary disease, but also in terms of increased non-coronary comorbidity with high prevalence of hypertension, diabetes, peripheral arterial disease, heart failure and a history of strokes.

Newer technology research is needed to facilitate and simplify the revascularization techniques and making them safer and more standardized and predictable. Ability of intervention cardiologist and center's experience had key role in achieving final success. Modern dedicated wires, balloons and retrograde technique are not used most of the time because of cost constrain and lack of experience in developing countries. Hence young cardiologist should have CTO revascularization training. CTO revascularization should not be avoided on the basis of "historical" poor success rates, unclear patient benefits, adverse outcomes or time and resource use.

\section{References}

1. KS Gopakumar, Iype Mathew, Viswanathan Sunitha, et al. The demographic and clinical profile of patients undergoing coronary chronic total occlusion (CTO) intervention in a teaching hospital in Kerala from the CTO investigators- Kerala (CTOI-K) Group. JMSCR. 2017;05(05):21881-21885.

2. Morino Y, Abe M, Morimoto T, Kimura T, Hayashi Y, Muramatsu T, Ochiai M, et al. Predicting successful guidewire crossing through chronic total occlusion of native coronary lesions within 30 minutes: the J-CTO (Multicenter CTO Registry in Japan) score as a difficulty grading and time assessment tool. JACC Cardiovasc Interv. 2011;4(2):213-221.

3. Bennett J, Kayaert P, Bataille Y, Dens J. Percutaneous coronary interventions of chronic total -occlusions; a review of clinical indications, treatment strategy and current practice. Acta Cardiol. 2017;72(4):357-369.

4. Artis Kalnins, Ieva Strele, Irena Kurcalte, et al. Chronic total coronary artery occlusion recanalization with percutaneous coronary intervention - single centre 10-year experience. Proc Latvian Acad Sci. 2018;72(712):1-8.

5. Christofferson RD, Lehmann KG, Martin GV, Every N, Caldwell JH, Kapadia SR. Effect of chronic total coronary occlusion on treatment strategy. Am J Cardiol. 2005;95(9):1088-1091.

6. Fefer P, Knudtson ML, Cheema AN, Galbraith PD, Osherov AB, Yalonetsky S, Gannot S, et al. Current perspectives on coronary chronic total occlusions: the Canadian Multicenter Chronic Total Occlusions Registry. J Am Coll Cardiol. 2012;59(11):991-997.

7. Grantham JA, Marso SP, Spertus J, House J, Holmes DR, Jr., Rutherford BD. Chronic total occlusion angioplasty in the United States. JACC Cardiovasc Interv. 2009;2(6):479-486.

8. Valenti R, Migliorini A, Signorini U, Vergara R, Parodi G, Carrabba N, Cerisano G, et al. Impact of complete revascularization with percutaneous coronary intervention on survival in patients with at least one chronic total occlusion. Eur Heart J. 2008;29(19):2336-2342.

9. Brilakis ES, Banerjee S, Karmpaliotis D, Lombardi WL, Tsai TT, Shunk KA, Kennedy KF, et al. Procedural outcomes of chronic total occlusion percutaneous coronary intervention: a report from the NCDR (National Cardiovascular Data Registry). JACC Cardiovasc Interv. 2015;8(2):245-253.

10. Yamamoto E, Natsuaki M, Morimoto T, Furukawa Y, Nakagawa Y, Ono K, Mitsudo K, et al. Long-term outcomes after percutaneous coronary intervention for chronic total occlusion (from the CREDO-Kyoto registry cohort-2). Am J Cardiol. 2013;112(6):767-774.

11. Srinivas VS, Brooks MM, Detre KM, King SB, 3rd, Jacobs AK, Johnston J, Williams DO. Contemporary percutaneous coronary intervention versus balloon angioplasty for multivessel coronary artery disease: a comparison of the National Heart, Lung and Blood Institute Dynamic Registry and the Bypass Angioplasty Revascularization Investigation (BARI) study. Circulation. 2002;106(13):1627-1633.

12. Kolh P, Windecker S, Alfonso F, Collet JP, Cremer J, Falk V, Filippatos G, et al. 2014 ESC/EACTS Guidelines on myocardial revascularization: the Task Force on Myocardial Revascularization of the European Society of Cardiology (ESC) and the European Association for CardioThoracic Surgery (EACTS). Developed with the special contribution of the European Association of Percutaneous Cardiovascular Interventions (EAPCI). Eur J Cardiothorac Surg. 2014;46(4):517-592. 